\title{
Chapitre VII
}

\section{EMISSIONS SPECTROSCOPIQUES}

\section{INTRODUCTION}

Au terme de ce long voyage, il est temps d'aborder l'un des aspects les plus fascinants de l'étude de l'ionosphère et de la thermosphère : leur rayonnement. D'où vient-il ? De la désexcitation des atomes, molécules ou ions. On a vu au cours des chapitres précédents qu'il existe trois sources distinctes d'excitation : l'absorption de photons solaires, les recombinaisons chimiques et les collisions avec des particules suprathermiques. Les rayonnements diurne et nocturne n'ont pas exactement les mêmes sources. Le rayonnement diurne est dû principalement à la conjonction des sources photonique et chimique. Dans ce cas, les électrons mis en jeu sont essentiellement des électrons thermiques ou des photoélectrons, d'énergie inférieure à environ $250 \mathrm{eV}$. Le rayonnement nocturne, quant à lui, est dû aux seules recombinaisons chimiques puisque, par définition, il n'y a pas de source de lumière. Les précipitations de particules donnent naissance au rayonnement auroral, les fascinantes aurores polaires.

Il ne s'agit pas ici de passer en revue toutes les émissions des trois spectres (diurne, nocturne, auroral) : des références pourront aider à les trouver. Mais il s'agit d'en donner les caractéristiques essentielles. De la mesure de ce rayonnement, il est possible de déduire les concentrations des émetteurs, qu'ils soient neutres ou ioniques. Ce rayonnement dépend également de la température neutre, qu'on peut parfois également inférer. Il en va de même de la température exosphérique. On peut également déduire la concentration électronique et les caractéristiques des précipitations ou du flux UV solaire. Ces paramètres conduisent à des informations géophysiques, au moyen des équations proposées dans cet ouvrage, comme par exemple la conductivité ionosphérique, qui dépend de la concentration électronique, ou encore le flux précipité de particules. En effet, l'intensité lumineuse dépend de la production d'excitation, elle même calculée par la formule $V-54$ du chapitre $V$. La mesure de l'excitation permet donc de remonter à la forme du flux stationnaire de particules et de là, au flux précipité. La mesure du décalage Doppler d'une raie lorsqu'un instrument pointe dans différentes directions conduit directement à la mesure de la vitesse de l'émetteur : c'est ainsi que sont déterminés les vents neutres évoqués au chapitre VI.

Il existe bien sûr des limitations instrumentales. Les expériences optiques au sol dépendent fortement de la météorologie: le moindre nuage fait écran aux rayonnements ionosphériques 
et thermosphériques. Ces expériences ne peuvent pas déterminer l'altitude de l'émission, et ne mesurent que l'intégrale des émissions le long de la ligne de visée, c'est à dire sur une colonne d'atmosphère. De fait, les paramètres géophysiques déduits des mesures sont également des grandeurs intégrées. L'estimation des flux de précipitations ou de photons se fait au moyen de rapports de raies, et ne donne qu'une idée approchée de la réalité. Enfin, la calibration des instruments optiques est d'une extrême difficulté. Les instruments embarqués ne connaissent pas les difficultés liées à la météorologie, et peuvent déterminer des profils d'émission. Mais leur mouvement leur rend impossible la mesure des variations temporelles, ou la séparation des caractères temporel et spatial lors des mesures. Ainsi, la complémentarité des instruments sol et espace est-elle indispensable pour la compréhension de notre environnement.

Dans tout ce chapitre, nous utiliserons l'unité conventionnelle pour la photométrie : le Rayleigh (R), défini comme la mesure de l'émission omnidirectionnelle dans une colonne de section unité, le long de la ligne de visée :

$1 R=10^{6}$ photons $\mathrm{cm}^{-2} \mathrm{~s}^{-1}$

VII-1

La mesure du rayonnement diurne est d'une extrêmement difficile. L'émission du Soleil est de $5010^{7}$ à $50010^{7}$ R.nm ${ }^{-1}$, tandis que la moyenne de l'intensité des raies atmosphériques est de $300 \mathrm{R}_{\mathrm{nm}}{ }^{-1}$ ! Il faut donc des instruments très sensibles, avec des filtres très puissants, ou ... faire les mesures en orbite ! Un exemple caractéristique de spectre diurne entre 120 et $900 \mathrm{~nm}$ est donné sur la figure VII-1. Il a été acquis à bord de la navette spatiale, lors du vol STS 74. Les principales raies mesurées sont nommément identifiées.

Un spectre nocturne entre 300 et $800 \mathrm{~nm}$ a été assemblé sur la figure VII-2. De même, les raies les plus intenses sont identifiées. On retrouve des caractéristiques communes aux deux spectres, et d'autres particulières. Plutôt que de les détailler longueur d'onde après longueur d'onde, nous avons choisi de discuter les raies d'émission principales des constituants de l'atmosphère.

Les schémas orthodoxes de la physique atomique et moléculaire recourent à des conventions qui permettent au coup d'œil averti de situer une longueur d'onde émise par rapport à une autre. Nous présentons plutôt, sur les figures suivantes, les émissions principales de façon à les retrouver aisément, même en n'étant pas spécialiste de physique du rayonnement. Les énergies sont celles des seuils d'excitation ou d'ionisation correspondantes.

\section{PRINCIPALES RAIES D'ÉMISSION DE L'OXYGÈNE ATOMIQUE}

Les désexcitations de l'oxygène atomique se trouvent sur la figure $V I I-3$. La figure $V I I-4$ en représente les états ionisés. La raie à $135,6 \mathrm{~nm}$ est l'une des raies les plus intenses du rayonnement diurne. L'usage désigne par «135,6 nm » le doublet à 135,6 et 135,8 nm. Même de jour, la source collisionnelle est plus importante que la source électromagnétique directe pour cette excitation. Les électrons en jeu sont cependant des électrons créés par photoionisation. Vers $110 \mathrm{~km}$, le rayonnement émis peut à son tour exciter l'oxygène moléculaire : il y a absorption résonante, ou encore extinction résonante. 

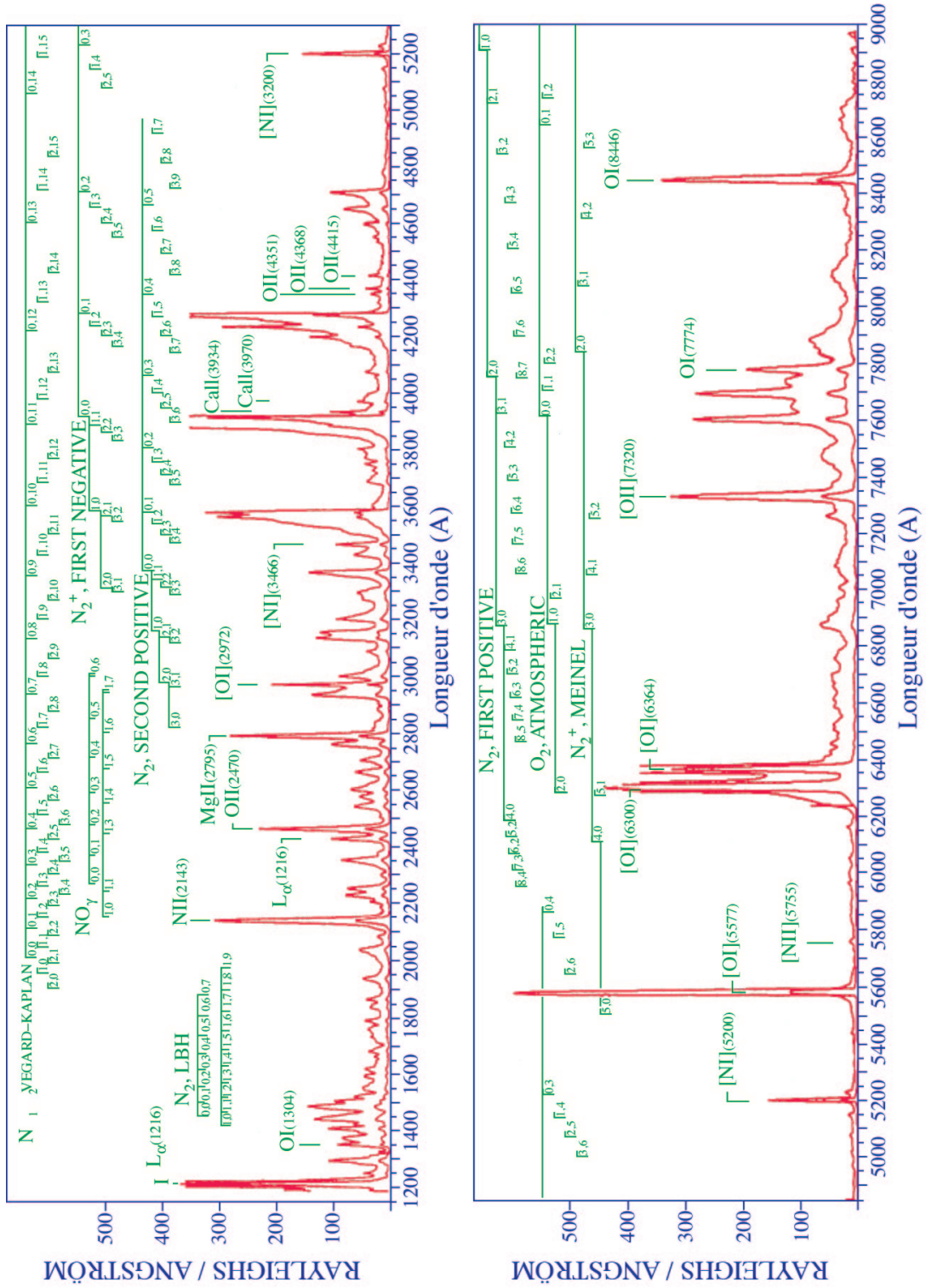

Figure VII-1 : ce spectre correspond au ciel diurne à moyenne latitude, pour des conditions solaires peu actives $\left(\mathbf{f}_{10.7}\right.$ de l'ordre de 80$)$ et un angle solaire zénithal de $48^{\circ}$ (Broadfoot et al., $\mathrm{N}_{2}$ triplet band systems and atomic oxygen in the dayglow, J. Geoph. Res., 102, 11567-11584, 1997) 


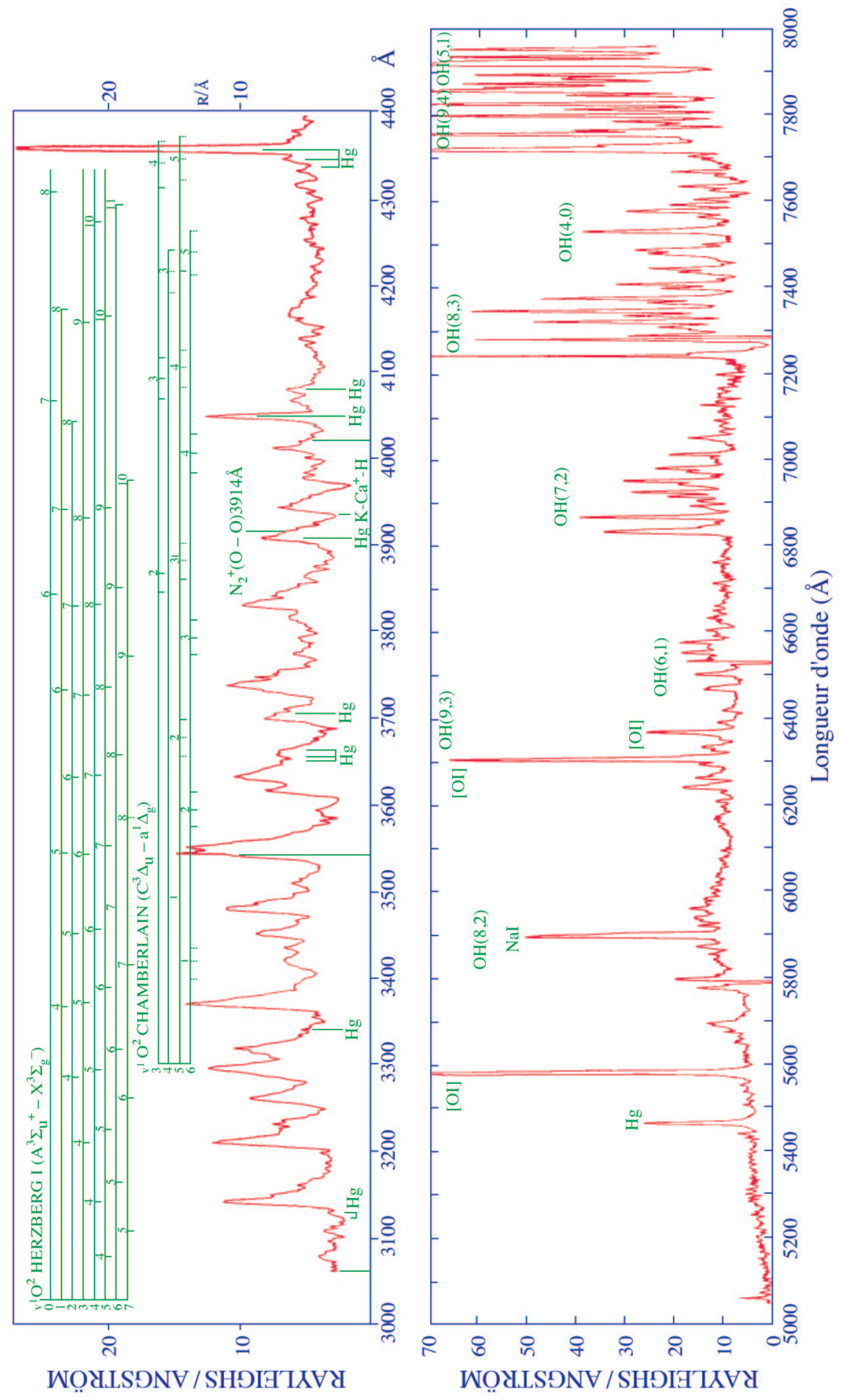

Figure VII-2 : spectre du ciel nocturne (L.A. Broadfoot et K.R Kendall, J. Geoph. Res., 73, 426, 1968 et autorisation de L.A. Broadfoot pour la figure du bas) 
On utilise aussi parfois le terme anglais quenching. L'origine de l'état ${ }^{5} \mathrm{~S}$ est l'excitation directe, ou la cascade depuis des états excités plus énergétiques (non figurés sur ce diagramme), et en particulier l'état ${ }^{5} \mathrm{P}$. Les profils d'émission de la raie à 135,6 $\mathrm{nm}$ acquis à bord de vaisseaux spatiaux ou de fusées donnent une mesure directe de la distribution de l'oxygène atomique et sont utilisés pour déduire la température exosphérique. La nuit, la source majeure de l'émission à $135,6 \mathrm{~nm}$ est la recombinaison chimique de $\mathrm{O}^{+}$avec un électron énergétique, qui laisse l'atome dans un état excité. La mesure de ce rayonnement donne alors une bonne idée de la densité électronique.

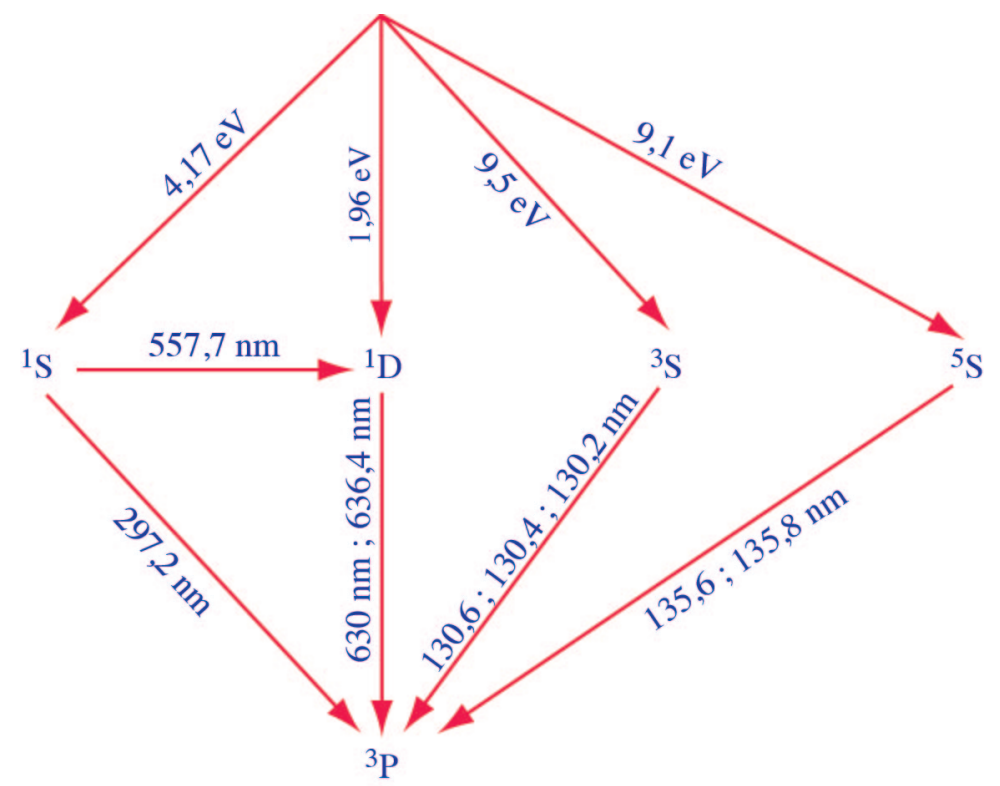

Figure VII-3 : principales voies d'excitation et de désexcitation de l'oxygène atomique

Au sommet du graphe, l'oxygène dans son état fondamental subit une collision ou absorbe un photon. Il est conduit vers un état excité. Le long des flèches qui mènent à l'état excité, nous avons fait figurer les seuils d'énergie. L'oxygène est rendu ensuite à l'état fondamental éventuellement via d'autres états excités par l'émission d'un photon, dont la longueur d'onde est notée le long de la flèche.

Le triplet à 130,4 nm (c'est à dire l'ensemble 130,2;130,4 et 130,6 nm) est également très intense. De jour, les sources collisionnelles et photoniques sont d'importances comparables. Les effets sont en revanche discernables : la source photonique produit un profil étroit, centré autour des trois raies, tandis que les collisions produisent un spectre si large qu'il ressemble à un continuum dans le spectre d'émission de l'oxygène. La nuit, c'est la recombinaison radiative qui devient le principal pourvoyeur de cette raie. L'état ${ }^{3} \mathrm{~S}$ est obtenu directement ou par cascade. La multiplicité des sources de cascade et les formes différentes de spectres font qu'en dépit de son intensité, cette raie d'émission est difficile à utiliser pour déduire la concentration de l'oxygène atomique. Une difficulté supplémentaire pour l'utilisation de cette raie est qu'elle entre en résonance avec une raie d'émission du Soleil. 


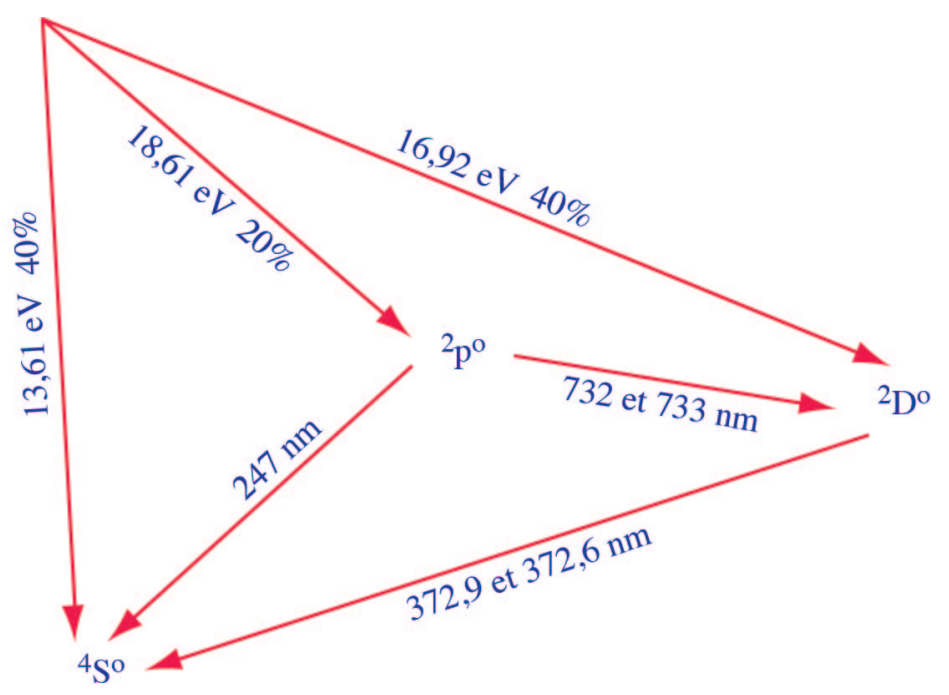

Figure VII-4 : principales voies d'ionisation de l'oxygène atomique

Les taux de chaque état sont également donnés le long de chaque flèche.

La raie d'émission de l'oxygène atomique la plus intense du rayonnement nocturne est la raie à $557,7 \mathrm{~nm}$, appelée également la raie verte, et produite principalement par la désexcitation de l'état ${ }^{l} \mathrm{~S}$ de l'oxygène, dont la durée de vie est $0,91 \mathrm{~s}$. Les principales réactions d'excitation de l'état ${ }^{l} \mathrm{~S}$ sont les suivantes :

La recombinaison dissociative est prépondérante en particulier sous $150 \mathrm{~km}$ :

$\mathrm{O}_{2}^{+}+e^{-} \rightarrow \mathrm{O}\left({ }^{3} \mathrm{P}\right)+\mathrm{O}\left({ }^{l} \mathrm{~S}\right)$

VII-2

La collision d'un atome d'oxygène avec de l'azote moléculaire excité est prépondérante en région $F$ :

$\mathrm{O}+\mathrm{N}_{2}\left(\mathrm{~A}^{3} \Sigma\right) \rightarrow \mathrm{N}_{2}+\mathrm{O}\left({ }^{l} \mathrm{~S}\right)$

VII-3

La collision de l'état fondamental avec un électron suprathermique est elle aussi une source importante :

$\mathrm{O}\left({ }^{3} \mathrm{P}\right)+e^{-} \rightarrow \mathrm{O}\left({ }^{1} \mathrm{~S}\right)+e^{-}$

VII-4

Dans ce cas, comme le montre le diagramme des excitations de l'oxygène atomique, l'électron doit avoir une énergie élevée : supérieure à $4,17 \mathrm{eV}$, et ne peut en aucun cas appartenir à la population thermique. Enfin, bien que de moindre importance, nous avons la recombinaison avec l'azote atomique :

$\mathrm{O}_{2}^{+}+\mathrm{N} \rightarrow \mathrm{NO}^{+}+\mathrm{O}\left({ }^{l} \mathrm{~S}\right)$

VII-5

L'absorption d'un photon dans la gamme 90-120 nm est le phénomène prépondérant vers $110 \mathrm{~km}$ :

$\mathrm{O}_{2}+\mathrm{hv} \rightarrow \mathrm{O}+\mathrm{O}\left({ }^{l} \mathrm{~S}\right)$ 
La chimie, à travers le processus de Barth, conduit à la production de $\mathrm{O}\left({ }^{1} \mathrm{~S}\right)$ en dessous de $120 \mathrm{~km}$. Ce phénomène devient même prépondérant sous $100 \mathrm{~km}$ :

$\mathrm{O}+\mathrm{O}+\mathrm{X} \rightarrow \mathrm{O}_{2}^{*}+\mathrm{X}$ VII-7

Immédiatement suivie de :

$\mathrm{O}_{2}{ }^{*}+\mathrm{O} \rightarrow \mathrm{O}_{2}+\mathrm{O}\left({ }^{l} \mathrm{~S}\right)$

Les puits de $\mathrm{O}\left({ }^{l} \mathrm{~S}\right)$ sont de deux ordres. En premier lieu, la collision avec un oxygène atomique ou moléculaire. Le second puit est l'émission spontanée d'un photon, à 557,7 nm ou à 297,2 $\mathrm{nm}$. Cette seconde émission est cependant 16 fois moins abondante que la première, la raie verte de l'oxygène.

L'intérêt de cette raie d'émission est qu'elle est relativement facile à mesurer et donne une bonne indication de la concentration de l'oxygène.

L'expérience a conduit à mettre en avant une émission bien particulière de l'oxygène atomique, vers $250 \mathrm{~km}$ : il s'agit de l'émission à $630 \mathrm{~nm}$ de l'oxygène excité dans l'état ${ }^{l} \mathrm{D}$, de durée de vie moyenne $110 \mathrm{~s}$. Nous allons l'examiner plus en détail que les précédentes.

Les sources principales sont les suivantes :

$\mathrm{O}_{2}^{+}+e^{-} \rightarrow \mathrm{O}\left({ }^{3} \mathrm{P}_{2,1}\right)+\mathrm{O}\left({ }^{l} \mathrm{D}\right)$

VII-9

Cette source est la plus importante la nuit, et la source prépondérante au-dessus d'environ $220 \mathrm{~km}$ le jour. Les électrons peuvent aussi bien être des photoélectrons que des électrons thermiques.

$\mathrm{O}_{2}+h \mathrm{v} \rightarrow \mathrm{O}\left({ }^{3} \mathrm{P}\right)+\mathrm{O}\left({ }^{l} \mathrm{D}\right)$

VII-10

... constitue la source prépondérante au-dessous de $160 \mathrm{~km}$, le jour bien sûr, puisqu'un photon est nécessaire. Dans ce cas, le photon est dans la gamme de Schumann - Runge, c'est à dire $135-175 \mathrm{~nm}$. Vers $180 \mathrm{~km}$, le jour, la majeure partie de $\mathrm{O}\left({ }^{l} \mathrm{D}\right)$ vient de :

$\mathrm{O}\left({ }^{3} \mathrm{P}\right)+e^{-} \rightarrow \mathrm{O}\left({ }^{l} \mathrm{D}\right)+e^{-}$

VII-11

L'électron incident doit avoir une énergie supérieure au seuil d'excitation, c'est à dire $1,96 \mathrm{eV}$.

$\mathrm{O}\left({ }^{l} \mathrm{~S}\right) \rightarrow \mathrm{O}\left({ }^{l} \mathrm{D}\right)+h v$

VII-12

Cette source est une source secondaire la nuit, puisque comme nous l'avons discuté cidessus, l'état ${ }^{l} \mathrm{~S}$ est dû, la nuit à la recombinaison de l'ion moléculaire d'oxygène. Le photon est émis à $557,7 \mathrm{~nm}$. Cette longueur d'onde est donc un marqueur de $\mathrm{O}\left({ }^{l} \mathrm{~S}\right)$ !

$\begin{array}{lr}\mathrm{N}\left({ }^{2} \mathrm{D}\right)+\mathrm{O} \rightarrow \mathrm{N}\left({ }^{4} \mathrm{~S}\right)+\mathrm{O}\left({ }^{l} \mathrm{D}\right) & \text { VII-13 } \\ \mathrm{N}^{+}+\mathrm{O}_{2} \rightarrow \mathrm{NO}^{+}+\mathrm{O}\left({ }^{l} \mathrm{D}\right) & \text { VII-14 } \\ \mathrm{N}\left({ }^{2} \mathrm{D}\right)+\mathrm{O}_{2} \rightarrow \mathrm{NO}+\mathrm{O}\left({ }^{l} \mathrm{D}\right) & \text { VII-15 }\end{array}$

Ces deux dernières réactions ne peuvent avoir lieu qu'aux altitudes où on trouve de l'azote atomique et de l'oxygène moléculaire, c'est à dire en région $E$. Néanmoins, l'azote atomique est un atome extrêmement minoritaire de la thermosphère, et cette réaction contribue très peu au rayonnement global de la raie rouge. 
Voyons à présent les puits. Il existe trois assombrissements par collision :

$\begin{array}{lr}\mathrm{O}\left({ }^{l} \mathrm{D}\right)+\mathrm{N}_{2} \rightarrow \mathrm{O}\left({ }^{3} \mathrm{P}\right)+\mathrm{N}_{2} & \text { VII-16 } \\ \mathrm{O}\left({ }^{l} \mathrm{D}\right)+\mathrm{O}_{2} \rightarrow \mathrm{O}\left({ }^{3} \mathrm{P}\right)+\mathrm{O}_{2} & \text { VII-17 }\end{array}$

Notons qu'il s'agit ici aussi d'un puits de région $E$.

$\mathrm{O}\left({ }^{1} \mathrm{D}\right)+\mathrm{O}\left({ }^{3} \mathrm{P}\right) \rightarrow \mathrm{O}\left({ }^{3} \mathrm{P}\right)+\mathrm{O}\left({ }^{3} \mathrm{P}\right)$

VII-18

L'oxygène se retrouve dans son état fondamental.

Pour l'ensemble des réactions ci-dessus, les coefficients sont sujets à débats. La bibliographie donnée en fin de chapitre permettra d'en trouver des expressions, dont une partie est donnée dans l'annexe II. Ils dépendent le plus souvent des températures (neutre, ionique, électronique).

Nous avons également trois désexcitations spontanées, qui constituent les trois puits principaux, et forment l'émission de la raie rouge de l'oxygène. Les photons ont des longueurs d'onde de $630 \mathrm{~nm}, 636,4 \mathrm{~nm}$ et $639,2 \mathrm{~nm}$.
$\mathrm{O}\left({ }^{l} \mathrm{D}\right) \rightarrow \mathrm{O}\left({ }^{3} \mathrm{P}\right)+h v$
$\mathrm{A}_{630}=7,110^{-3} \mathrm{~s}^{-1}$
VII-19
$\mathrm{O}\left({ }^{l} \mathrm{D}\right) \rightarrow \mathrm{O}\left({ }^{3} \mathrm{P}\right)+h v$
$\mathrm{A}_{636,4}=2,210^{-3} \mathrm{~s}^{-1}$
VII-20
$\mathrm{O}\left({ }^{l} \mathrm{D}\right) \rightarrow \mathrm{O}\left({ }^{3} \mathrm{P}\right)+h v$
$\mathrm{A}_{639,2}=2,7410^{-4} \mathrm{~s}^{-1}$
VII-21

Les coefficients A sont les coefficients d'Einstein, définis au chapitre $I V$. L'état le plus abondant est le premier, de durée de vie $140 \mathrm{~s}$. C'est l'état métastable. Ce triplet émet dans le rouge. On pourra juger de l'importance de cette émission, sur la photographie VII-5, prise avec un appareil photographique classique, par le spationaute Jean Loup Chrétien lors de son vol du 24 juin au 2 juillet 1982 à bord du vaisseaux SOYOUZ T6 et de la station SALIOUT 7. On y voit sans peine la raie rouge de l'oxygène dans la région $F$.

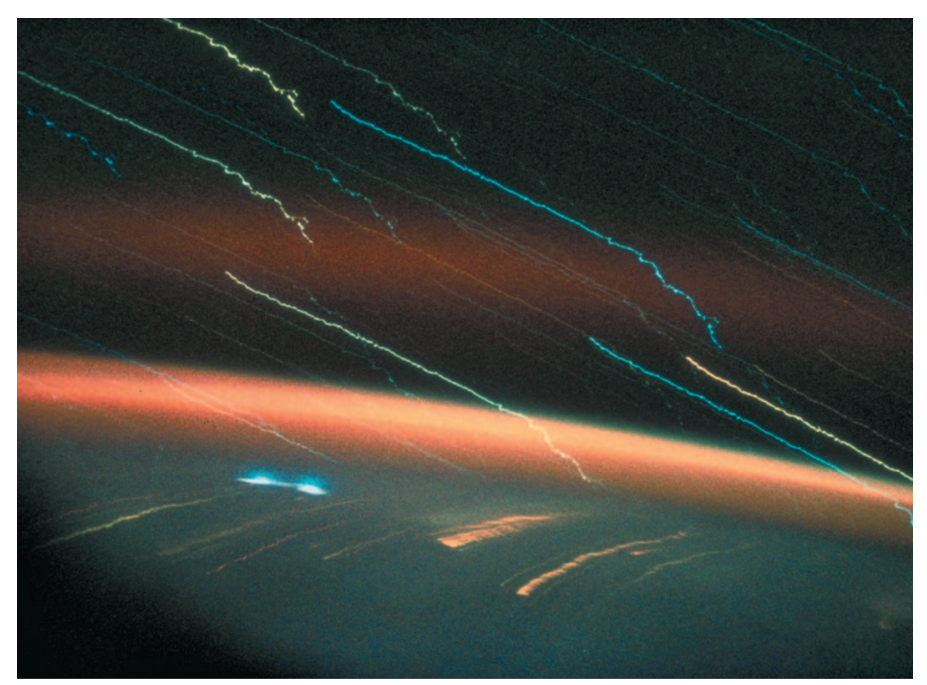

Figure $V I I-5$ : émission de la raie rouge vers $250 \mathrm{~km}$

Les traits bleus et blancs sont les étoiles qui semblent défiler pendant la prise de vue. En bas, le limbe de la Terre (source : S. Koutchmy, IAP-CNRS) 
Concernant l'ionisation, les ions excités ont une énergie de $5 \mathrm{eV}$ dans l'état ${ }^{2} \mathrm{P}^{\circ}$ et de $3,31 \mathrm{eV}$ dans l'état ${ }^{2} \mathrm{D}^{\circ}$. Ces sources sont importantes dans les aurores polaires, l'ionisation étant alors due aux impacts électroniques. L'état ${ }^{2} \mathrm{P}^{\circ}$ a une durée de vie de $5 \mathrm{~s}$, mais l'état ${ }^{2} \mathrm{D}^{\circ}$ a une durée de vie de $3,6 \mathrm{~h}$ !

La figure VII-6 montre le résultat d'une modélisation qui permet de visualiser les importances relatives des diverses sources d'excitation de la raie rouge. Les trois courbes en bleu en haut de cette figure montrent les densités électroniques et les températures électroniques et ioniques le 21 juillet 1993 au dessus du radar EISCAT (à $62^{\circ}$ de latitude nord), vers midi. Le flux décimétrique vaut 106 , et l'indice Ap est égal à 14 . Les croix correspondent aux valeurs mesurées, et les traits sont le résultat d'une modélisation. Les équations résolues sont celles du chapitre $V$ donnant accès aux productions primaires et secondaires, aux flux de chaleur, et aux trois grandeurs macroscopiques mesurées. L'atmosphère neutre est décrite au chapitre $I I$.

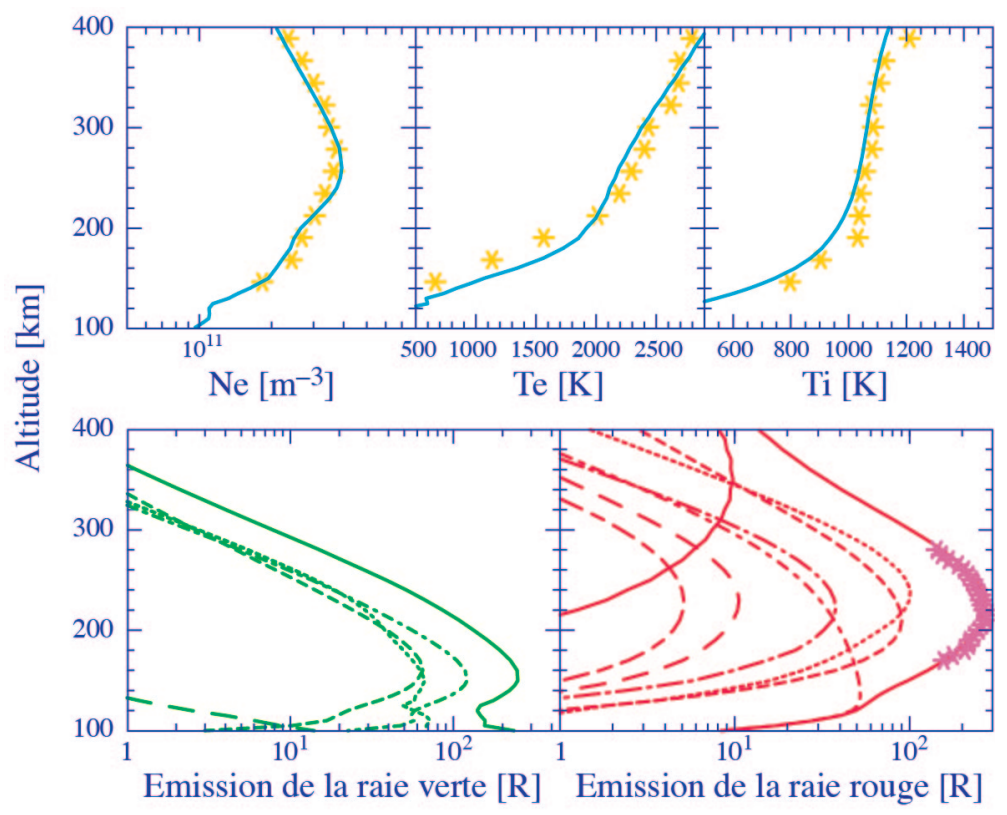

Figure VII-6 : en haut, mesure et calcul des paramètres ionosphériques, en bas, calcul et mesure des émissions de l'oxygène atomique

(O. Witasse, thèse de troisième cycle de l'UJF, 1998)

Les courbes du bas représentent l'émission de la raie verte et celle de la raie rouge. A $140 \mathrm{~km}$ par exemple, la source principale d'excitation de la raie verte est la collision avec l'azote moléculaire (réaction VII-2), puis respectivement la recombinaison avec l'ion moléculaire oxygène (réaction VII-3), la collision entre les électrons et l'oxygène atomique (réaction $V I I-4$ ) et enfin entre l'ion moléculaire oxygène et l'azote atomique (réaction VII-5). Les sources de la raie rouge se distinguent bien à $240 \mathrm{~km}$. Par ordre d'importance décroissante, nous y trouvons la recombinaison électronique avec l'ion moléculaire oxygène (réaction VII-9), la collision entre l'oxygène atomique et des électrons suprathermiques 
(réaction VII-11), la collision entre l'ion moléculaire oxygène et l'azote atomique (réaction $V I I-15$ ), la photoexcitation (réaction $V I I-10$ ), la désexcitation de la raie verte (réaction VII-12), la collision entre l'ion atomique d'azote et la molécule d'oxygène (réaction $V I I-14)$ et enfin la collision entre l'oxygène atomique et des électron thermiques (réaction VII-11).

Au moment de cette mesure, le satellite UARS mesurait l'émission de la raie rouge le long de l'axe de visée du radar. Cette mesure est montrée avec des croix, et permet d'une certaine manière de valider la discussion ci-dessus. On constate que la raie rouge est maximale en région $F$, vers $230 \mathrm{~km}$, avec des taux d'émissions de l'ordre de $230 \mathrm{R}$.

\section{PRINCIPALES RAIES D'ÉMISSION DE L'OXYGÈNE MOLÉCULAIRE}

Nous n'allons passer en revue que les émissions les plus utiles pour l'aéronomie. Les figures $V I I-7$ et $V I I-8$ présentent, dans le même format que pour l'oxygène atomique, les émissions principales de l'oxygène moléculaire. Nous avons à présent à traiter avec une molécule, et donc avec des bandes et systèmes d'excitations qui incluent de nombreuses excitations vibrationnelles (étudiées dans le chapitre $V$ ). Les transitions portent fréquemment le nom de leur découvreur, ou un nom conventionnel. Ces noms sont écrits auprès des flèches correspondantes.

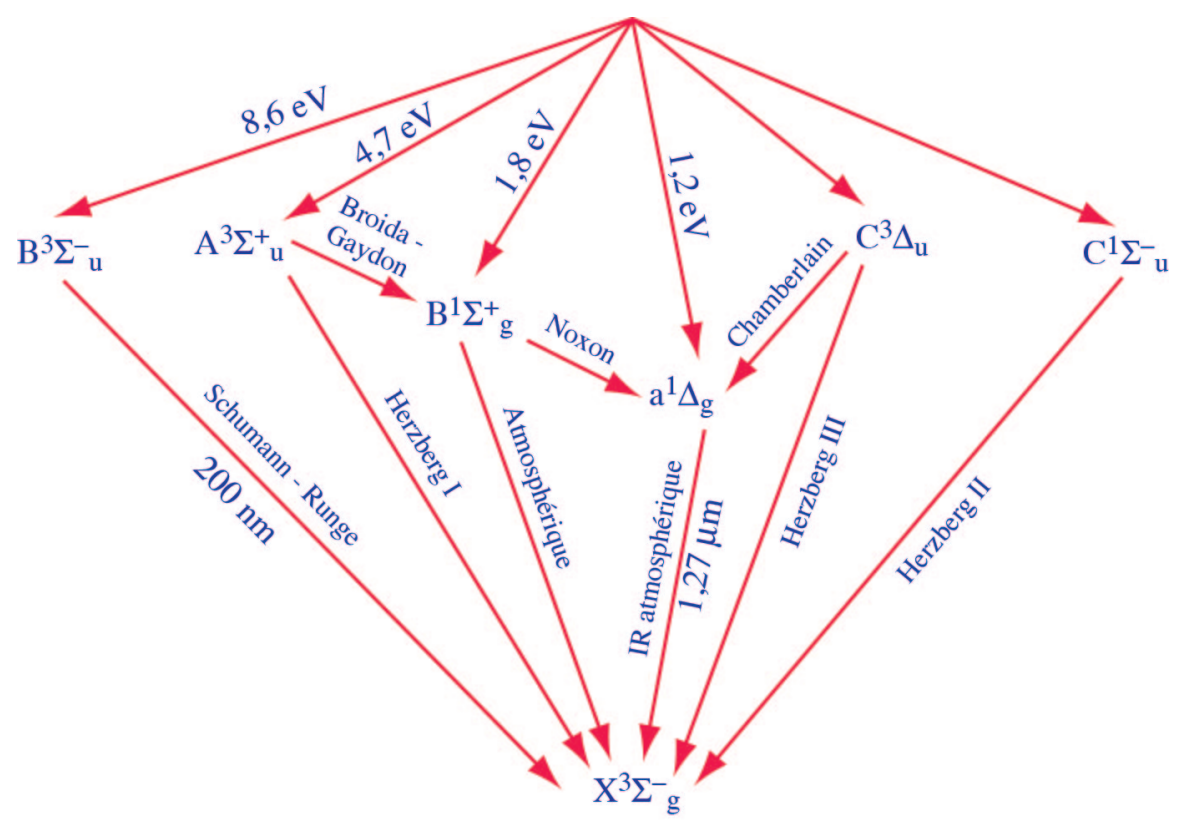

Figure VII-7 : excitations de l'oxygène moléculaire

La norme de ce diagramme est la même que pour l'oxygène atomique.

Nous avons fait figurer, en outre, les noms des bandes d'émissions. 
Il saute aux yeux, devant le spectre de rayonnement nocturne, que toute la région de 300 à $440 \mathrm{~nm}$ est dominée par deux systèmes : celui de Herzberg et celui de Chamberlain. L'excitation de l'oxygène moléculaire dans l'état $\mathrm{A}^{3} \Sigma_{u}^{+}$vient de la collision à trois corps entre deux atomes d'oxygène et un troisième corps, molécule ou atome. La transition qui va de l'état $\mathrm{A}^{3} \Sigma_{u}^{+}$vers le fondamental viole la règle que nous avions énoncée au chapitre $I V$ selon laquelle on ne peut pas aller d'un état $\Sigma^{+}$vers un état $\Sigma^{-}$. Les atomes d'oxygène viennent de la dissociation d'oxygène moléculaire, qui diffusent vers le bas dans l'atmosphère, là où des réactions à trois corps deviennent plus faciles à mettre en jeu (vers $100 \mathrm{~km}$ ). Le système de Chamberlain résulte lui aussi d'une réaction à trois corps similaire.

Le système de Schumann-Runge est un absorbant important de l'ultraviolet lointain dans l'atmosphère moyenne. Il émet dans un quasi continuum entre 125 et $175 \mathrm{~nm}$. Le rayonnement émis excite de façon différente les diverses raies de la bande $L B H$ de l'azote moléculaire, si bien que le rapport des raies de l'azote devient un indicateur de la concentration de l'oxygène moléculaire!

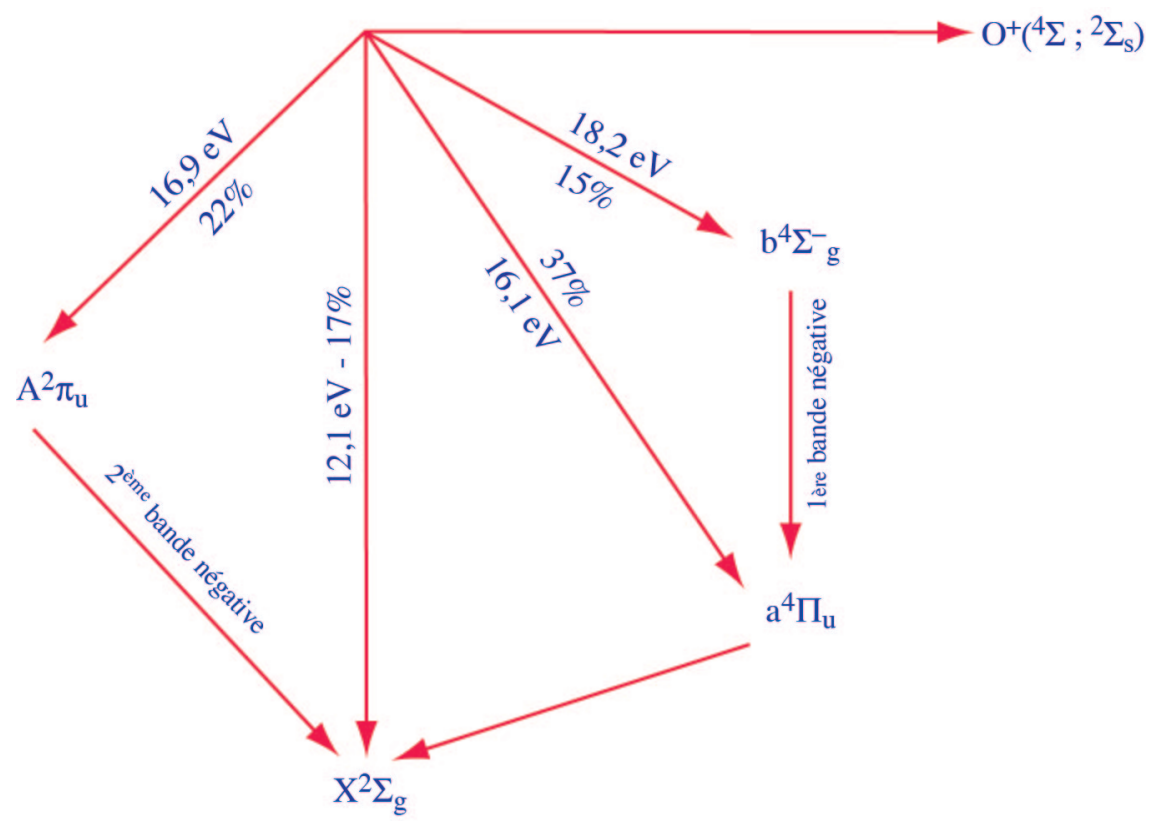

Figure VII-8 : ionisations de l'oxygène moléculaire

\section{PRINCIPALES RAIES D'ÉMISSION DE L'AZOTE MOLÉCULAIRE}

De très nombreux paramètres de l'atmosphère sont déduits de la mesure du rayonnement de $\mathrm{N}_{2}$. Les figures $V I I-9$ et $V I I-10$ présentent, dans le même format que pour l'oxygène 
atomique, les émissions principales de l'azote moléculaire. La figure VII-11 montre en plus les ionisations dissociatives. L'état fondamental de l'azote moléculaire est l'état $\mathrm{X}^{l} \Sigma_{g}^{+}$, et celui de l'ion $\mathrm{N}_{2}^{+}$est $\mathrm{X}^{2} \Sigma_{g}^{+}$. Enfin, nous avons fait figurer les gammes spectrales des émissions, IR signifiant comme d'habitude l'infrarouge, et UV l'ultraviolet. Ici encore, nous n'en discuterons que les raies les plus importantes. Un caractère particulier du spectre d'excitation est la cascade qui engendre la seconde bande positive, la première, puis la bande de Vegard-Kaplan. On appelle parfois abusivement cette cascade le triplet de l'azote moléculaire.

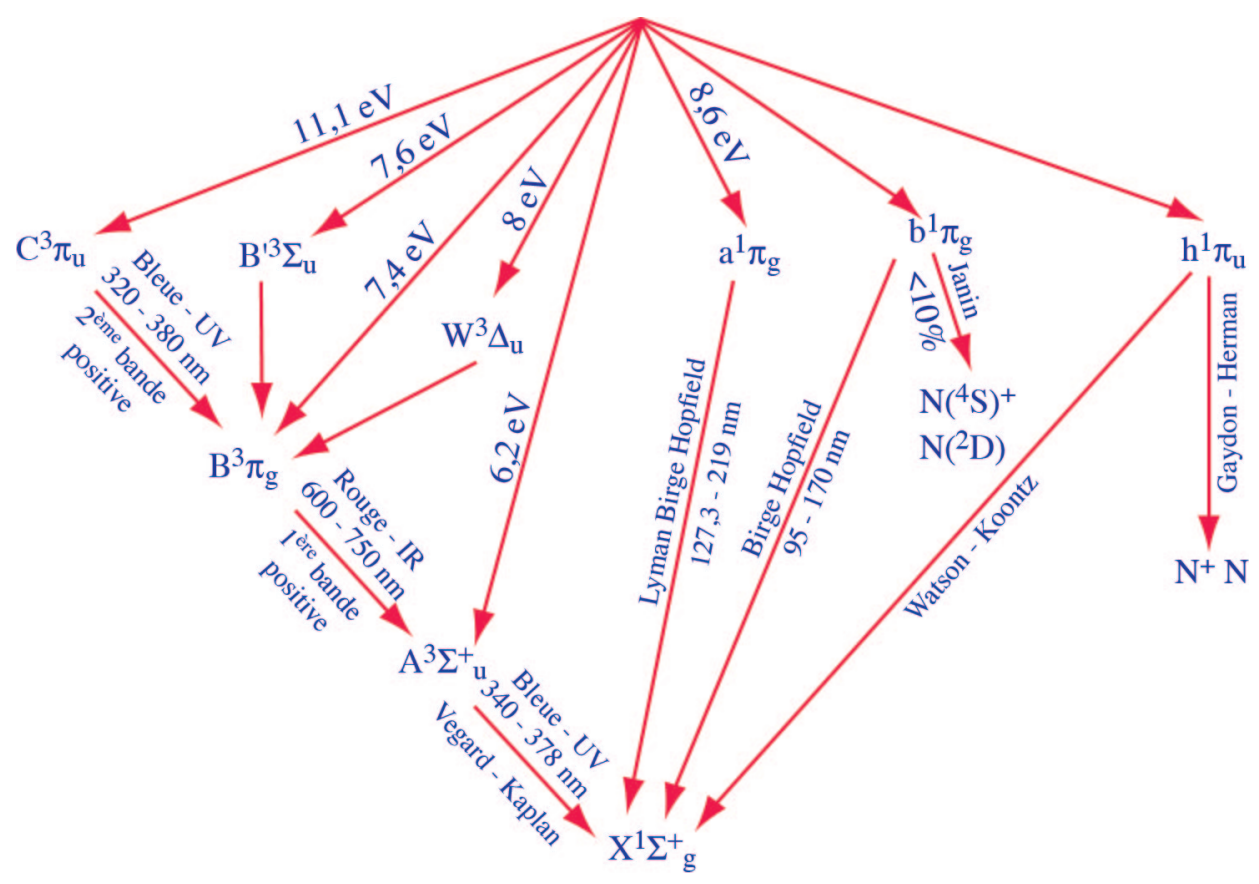

Figure VII-9 : excitations de l'azote moléculaire

L'état $\mathrm{C}^{3} \Pi_{u}$ a un temps de vie court, de l'ordre de $10^{-7} \mathrm{~s}$. Il possède une seule source, qui est la collision d'une molécule d'azote avec un électron énergétique, et un seul puits, la désexcitation vers l'état $\mathrm{B}^{3} \Pi_{g}$. Cette caractéristique en fait un traceur idéal du taux d'excitation par collision électronique et partant, des caractéristiques des précipitations. La seconde bande positive possède des raies entre 320 et $380 \mathrm{~nm}$, mais $86 \%$ de la population $\mathrm{C}^{3} \Pi_{u}$ est dans l'état $v=0$ ou 1 . D'autre part, $49 \%$ de l'état $v^{\prime}=0$ se désexcite en émettant à $337,1 \mathrm{~nm}$, qui est l'une des raies intenses de ce système dans le spectre nocturne. Elle est donc utilisée pour connaître les caractéristiques des photoélectrons ou le profil de concentration de $\mathrm{N}_{2}$. Le rayonnement de la seconde bande positive se fait dans le bleu et l'ultraviolet. Comme l'altitude du pic de production de la seconde bande positive dépend de la concentration, et celle ci de la température, on tire en particulier de son observation la température exosphérique. 


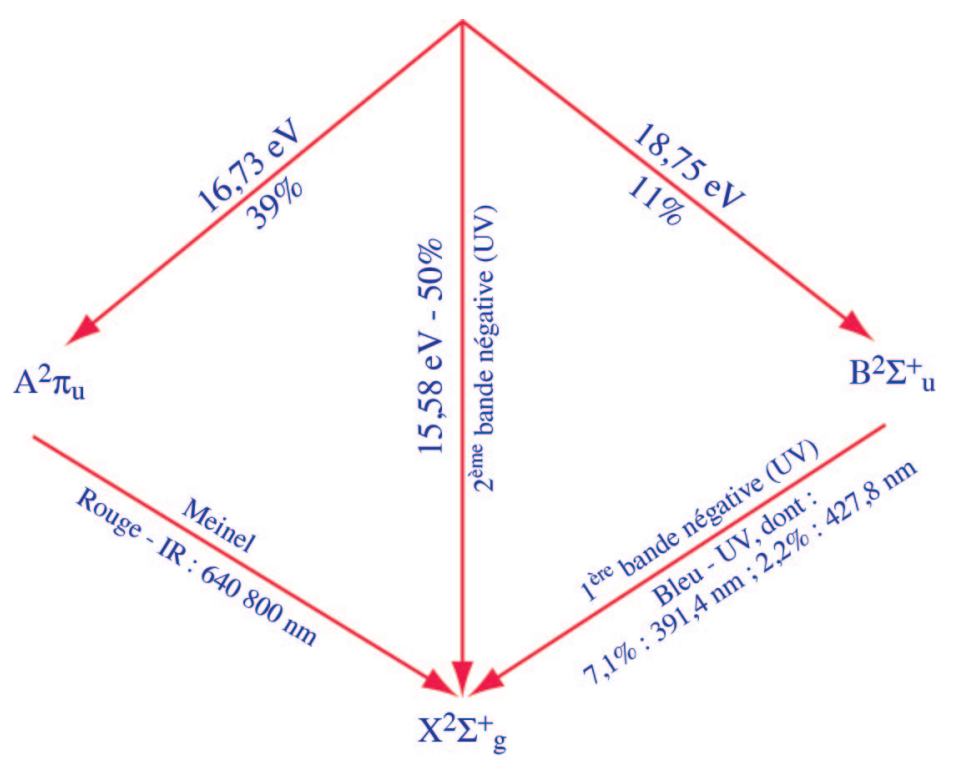

Figure VII-10 : ionisations de l'azote moléculaire

La première bande positive résulte de la désexcitation de l'état $\mathrm{B}^{3} \Pi_{g}$. Cependant, les sources de cet état sont multiples. Outre $\mathrm{C}^{3} \Pi_{u}$ déjà cité, nous avons fait figurer sur ce diagramme les états $\mathrm{B}^{3} \Sigma_{u}, \mathrm{~W}^{3} \Delta_{u}$ et la production directe par collision. Il existe d'autres sources, d'importance moindre : $\mathrm{D}^{3} \Sigma_{u}^{+}$et $\mathrm{E}^{3} \Sigma_{g}^{+}$. La première bande positive est très intense, mais divisée en de nombreux niveaux excités sur une large bande spectrale. Elle constitue un acteur important de l'analyse du rayonnement diurne, et donne un outil de mesure du taux total d'excitation par collisions électroniques. Elle émet principalement entre 600 et $750 \mathrm{~nm}$, c'est à dire dans le rouge.

Continuant cette cascade, nous arrivons à la bande Vegard-Kaplan, dominante dans le spectre diurne. Elle résulte de la désexcitation de l'état $\mathrm{A}^{3} \Sigma_{u}^{+}$vers le fondamental. Cet état excité possède un temps de vie de l'ordre de $2 \mathrm{~s}$, assez long pour autoriser la diffusion résonnante de la lumière solaire à travers la bande première positive et l'assombrissement par l'oxygène. L'observation de Vegard-Kaplan est utile pour contrôler cet assombrissement. C'est une bande intense parce presque tous les états cités ci-dessus y mènent. Les impacts directs peuplent des états vibrationnels de haut niveau, et ne contribuent pas de façon substantielle aux émissions observables. Cette bande est un bon indicateur de la température neutre locale. Elle rayonne dans le bleu / violet.

Quittant cette cascade, la bande Lyman-Birge-Hopfield, ou LBH est une raie importante durant la journée, issue d'impacts électroniques. Cette bande est elle aussi un bon marqueur des photoélectrons, en particulier dans la gamme 10 à $50 \mathrm{eV}$, et de la température exosphérique. La bande BH (Birge-Hopfield) est excitée par des électrons de plus haute énergie, et devient témoin de la population vibrationnelle de l'état fondamental. Les bandes $L B H$ et $B H$ ont été également observées sur Titan, satellite de Saturne. 


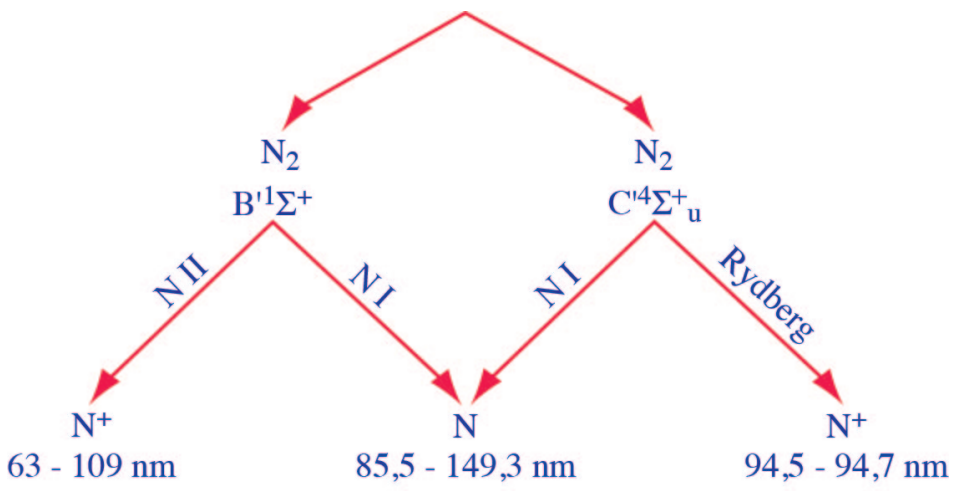

\section{Figure VII-11 : ionisations dissociatives de l'azote moléculaire}

Dans le schéma correspondant à l'ionisation, et parmi les $11 \%$ qui mènent à l'état $\mathrm{B}^{2} \Sigma_{u}^{+}$, $64,5 \%$ produisent une émission à $391,4 \mathrm{~nm}$, tandis que les $35,5 \%$ restant produisent une émission à $427,8 \mathrm{~nm}$. La première bande négative, et en particulier le rayonnement à $391,4 \mathrm{~nm}$, est un indicateur précieux de la «dureté » des précipitations, source principale de l'état $\mathrm{B}^{2} \Sigma_{u}^{+}$. Comme ce rayonnement est sensiblement proportionnel à la concentration d'ions moléculaires d'azote, on obtient en outre par la mesure du profil de la raie le profil de concentration cet ion. Cette bande domine la région bleue du spectre.

\section{ESPÈCES MINORITAIRES}

Les spectres diurnes et nocturnes exhibent aussi des raies dues à d'autres espèces que l'oxygène ou l'azote. La nuit, la molécule de monoxyde d'azote produit une émission entre 160 et $290 \mathrm{~nm}$ due aux états vibrationnels de deux systèmes. Les états excités sont issus de l'association réversible d'un atome d'oxygène avec un atome d'azote. Toute la partie du spectre nocturne située dans le visible possède également un fond continu d'émissions de $\mathrm{NO}$ et $\mathrm{NO}_{2}$, émis à environ $100 \mathrm{~km}$. Dans ce cas, une molécule de monoxyde d'azote acrète un atome d'oxygène pour former une molécule excitée de dioxyde d'azote. Celle-ci se désexcite en émettant de la lumière $(>400 \mathrm{~nm})$, et se casse lors d'une collision avec $\mathrm{O}$ pour redonner $\mathrm{NO}$ et $\mathrm{O}_{2}$. NO est à l'origine d'un système important également entre 200 et $280 \mathrm{~nm}$. Il s'agit de la bande nommée $\gamma$, qui correspond à la désexcitation de l'état $\mathrm{X}_{2} \Pi$ vers l'état $\mathrm{A}^{2} \Sigma^{+}$. Cette émission est utilisée pour connaître l'abondance de NO dans la thermosphère. Cette mesure a permis de déterminer que la concentration de NO vers $110 \mathrm{~km}$ varie avec l'activité solaire et témoigne à la fois de la rotation du Soleil et de son cycle à 11 ans.

L'azote atomique, neutre ou ionisé, produit un rayonnement dans le proche UV, ainsi que plusieurs raies dans l'UV lointain $(149,3 ; 120$ et $113,5 \mathrm{~nm})$. Les états excités sont dus à l'absorption de photons ou à des collisions avec des photoélectrons soit par l'atome, soit par la molécule d'azote, qui se dissocie au cours de la réaction. 
Les rayonnements Lyman $\alpha(121,6 \mathrm{~nm})$ et Lyman $\beta(102,6 \mathrm{~nm})$ de l'hydrogène sont issus de la diffusion résonnante de la radiation solaire par les atomes de la couronne atmosphérique terrestre : l'hydrogène terrestre absorbe puis réémet le rayonnement Lyman solaire. Elles sont bien visibles sur les images de Dynamics Explorer que nous montrons dans le chapitre consacré à la magnétosphère. La couronne que dessine ce rayonnement est appelée la géocouronne. En effet, l'hydrogène et l'hélium, espèces mineures de la thermosphère, deviennent prépondérantes dans l'exosphère. L'observation de la raie Lyman $\alpha$ est utile pour déterminer la variation du flux solaire ou la concentration de l'hydrogène. De même, le rayonnement de diffusion résonnante de l'hélium $(58,4$ et $53,7 \mathrm{~nm})$ sert à en déterminer l'abondance. Néanmoins, la raie à $53,7 \mathrm{~nm}$ est considérablement contaminée par l'émission de l'oxygène atomique ionisé à 53,8 et $53,9 \mathrm{~nm}$.

Notons enfin plusieurs raies d'intensité diverses, due à la contamination anthropogénique au mercure ...

\section{LE RAYONNEMENT AURORAL}

Le rayonnement auroral est celui de l'ovale auroral. Les précipitations de particules se font sur une large gamme d'énergies : de quelques dizaines d'eV à plusieurs centaines de $\mathrm{keV}$. Les électrons secondaires permettent de peupler les basses énergies. Autant dire que la plupart des émissions décrites ci-dessus existent dans l'ovale auroral, mais que leur intensité peut y être considérablement augmentée, au point que le rayonnement visible devient parfaitement détectable à l'oeil nu : on a créé une aurore polaire, boréale au nord, australe au sud. Lors de précipitations « dures », c'est à dire d'énergies supérieures au keV, les collisions se font dans la basse région $F$ ou dans la région $E$. La seconde bande positive de l'azote moléculaire devient alors une émission dominante. L'aurore se teinte de bleu et de mauve. Si les précipitations ont des énergies de quelques centaines d'électronvolts, les excitations se font à plus haute altitude, et on obtient des aurores vertes. Des faisceaux de haute énergie très collimatés produisent des aurores de couleur très uniforme. Le ciel peut alors se nimber de rouge.

Il est très difficile de décrire les aurores. De vrais écrivains s'y sont essayé, auxquels nous n'aurons pas la prétention de nous confronter! On ne peut les voir que la nuit, car si l'ovale auroral existe en permanence, leur lumière est si ténue qu'elle est écrasée par celle du jour, et même par celle de la pleine lune. Comme elles se produisent au dessus de $80 \mathrm{~km}$, il faut également un ciel clair pour les voir. Ces conditions sont réunies en particulier en hiver, par les grands froids secs, ce qui a fait associer à tort les aurores avec l'arrivée du froid. Souvent, le phénomène commence par des faisceaux de lumière blanche pâle qui semblent tomber vers la Terre. Le phénomène s'intensifie en une quinzaine de minutes; il prend l'allure d'un voile qui oscillerait sous l'effet d'un vent hypothétique. En s'intensifiant, l'aurore se diapre de rouge en bas du voile, qui parfois frise le mauve. Plus en altitude, on distingue des franges de vert. A chaque instant se crée une nouvelle composition de couleurs, dans un ballet silencieux. En période de forte activité solaire, les aurores peuvent 
se succéder chaque nuit, alternant les tableaux : aurores diffuses, d'un vert dont les mouvements semblent imperceptibles, aurore rouge, grands arcs aux couleurs laiteuses homogènes, aurores rayées, dans lesquelles des stries verticales de couleurs alternent avec des zones d'ombre... Dans tous les cas, l'intensité lumineuse est si faible qu'on peut voir les étoiles les plus brillantes au travers. Leur longueur est très changeante, pouvant s'étaler jusqu'à la centaine de kilomètres, et leur durée varie de quelques minutes à quelques heures.

Les aurores polaires ont été la source de très nombreuses croyances des peuples des hautes latitudes. La plupart d'entre elles sont empruntes de terreurs. Par exemple, les Norvégiens interdisaient récemment encore aux enfants d'agiter un linge blanc (mouchoir, ...) devant une aurore, de crainte que celle-ci ne vienne capturer l'enfant pour l'emmener dans le ciel. A l'autre bout de la Terre, les Kurnais australiens frappaient l'aurore avec une «main morte », prise sur un proche décédé.

La première tentative d'interprétation scientifique date du philosophe grec Anaximène, vers 600 avant J.C. Il suggéra que l'air s'élevant de la Terre, se condense pour former des nuages puis, plus haut, se raréfie et brûle pour donner les aurores. Cette interprétation, faiblement modifiée, subsitera jusqu'au $17^{\mathrm{e}}$ siècle. A la fin du $19^{\mathrm{e}}$, les connaissances se sont un peu améliorées sur ces phénomènes. Camille Flammarion, dans son Astronomie Populaire, peut ainsi écrire en 1891 : «Le magnétisme terrestre a ses pôles, ses méridiens, son équateur, qui se déplacent sur et dans notre globe. Notre planète est en réalité un vaste aimant d'une puissance énorme, que Gauss a évaluée à celle de 8464 trillions de barres d'acier pesant chacune une livre et aimantées à saturation. »

Citant alors les observations de Carrington (voir chapitre $V$ ), il conclut :

« Il est très probable, presque certain, que cette correspondance entre l'état du Soleil et le magnétisme terrestre n'est pas fortuite mais réelle, et qu'il y a un rapport magnétique entre le Soleil et la Terre.

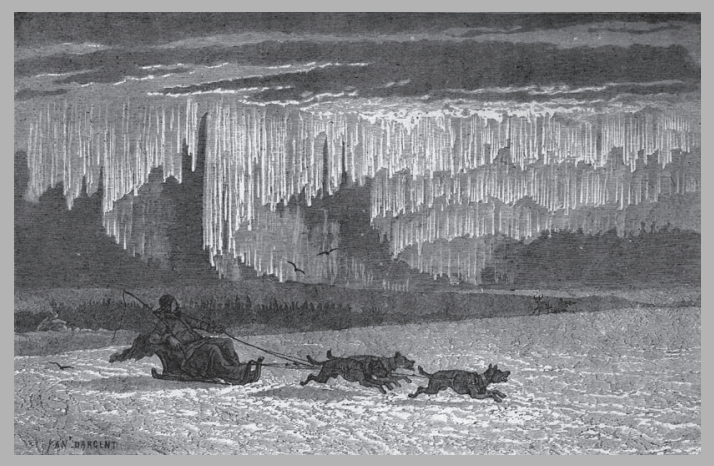

Figure VII-12 : cette gravure du $19^{\mathrm{e}}$ siècle portait comme légende : « la glace se soulevant sous une aurore boréale »

(Le pôle nord et le pôle sud, V. Tissot et C. Améro, Firmin didot, 1891) 
Cette correspondance paraît s'étendre aux aurores boréales. Le premier fait a été mis en évidence par Sabine, Wolf et Gautier ; celui-ci par Loomis et Zoellner. Le nombre et la grandeur des aurores visibles chaque année varie sur une période de onze ans, le maximum coïncidant avec celui des taches et des éruptions solaires ... On se souvient qu'autrefois, Arago se vantait de deviner une aurore visible en Suède et en Norvège par la seule inspection de l'aiguille aimantée à Paris. Il n'est donc pas surprenant que la correspondance remarquée entre l'état du Soleil et la boussole s'étende aux aurores ». Les statistiques, faites à partir des données du satellite de défense américain DMSP présentées au chapitre $V I$, ont montré qu'à $60^{\circ}$ de latitude nord, vers 20 heures locales, les particules précipitées sont le plus souvent d'énergie inférieure à $600 \mathrm{eV}$. Elles excitent l'hydrogène des hautes altitudes, qui retourne à son état fondamental par des émissions non visibles, et l'oxygène atomique, qui émet alors une lumière verte. Ce sont les aurores diffuses. En revanche, vers 21 heures et 6 heures locales, leur énergie - typiquement 1 à $10 \mathrm{keV}$ - leur permet de descendre plus bas dans l'atmosphère (jusqu'à $80 \mathrm{~km}$ ). Les ions, atomes et molécules excités se désexcitent en émettant beaucoup de lumière visible : du bleu foncé, du pourpre, et du bleu émeraude pour l'ion azote moléculaire, du vert et du blanc pour la molécule d'azote, du jaune pour l'oxygène moléculaire, du vert et du rouge pour l'oxygène atomique ... C'est le phénomène d'aurore polaire discrète.

La dynamique des aurores est impressionnante, et impossible à rendre au seul moyen de photographies. Des tourbillons se créent en quelques dixièmes de secondes, se propagent sur des distances de plusieurs dizaines de kilomètres en l'espace de quelques secondes. Il existe une nomenclature des aurores, qui va jusqu'aux aurores noires, qui sont les zones du ciel entre deux arcs auroraux. Cette nomenclature dépasse le cadre de ce livre.

Les photographies d'aurores rouges suivantes ont été prises par Kjell Raastad, en Norvège.
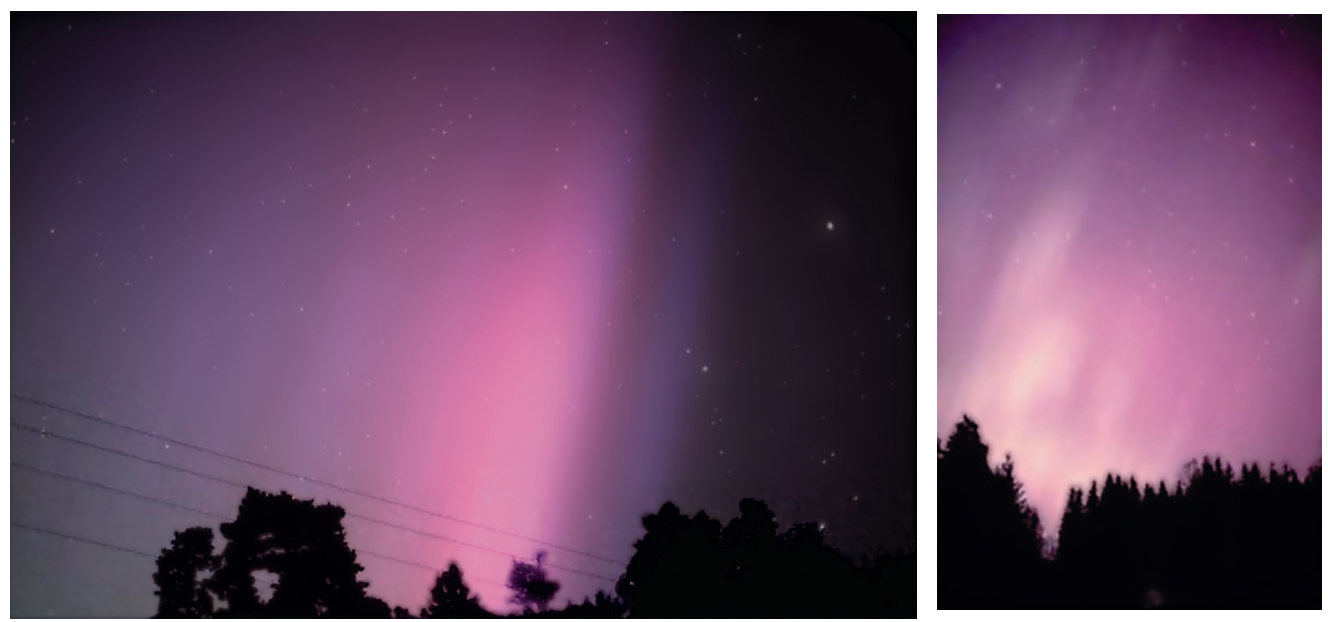
Quelques photographies d'aurores bleues et vertes, prises par Jyrki Manninen, de l'observatoire géophysique de Sodankylä, en Finlande, en montrent la diversité de formes.
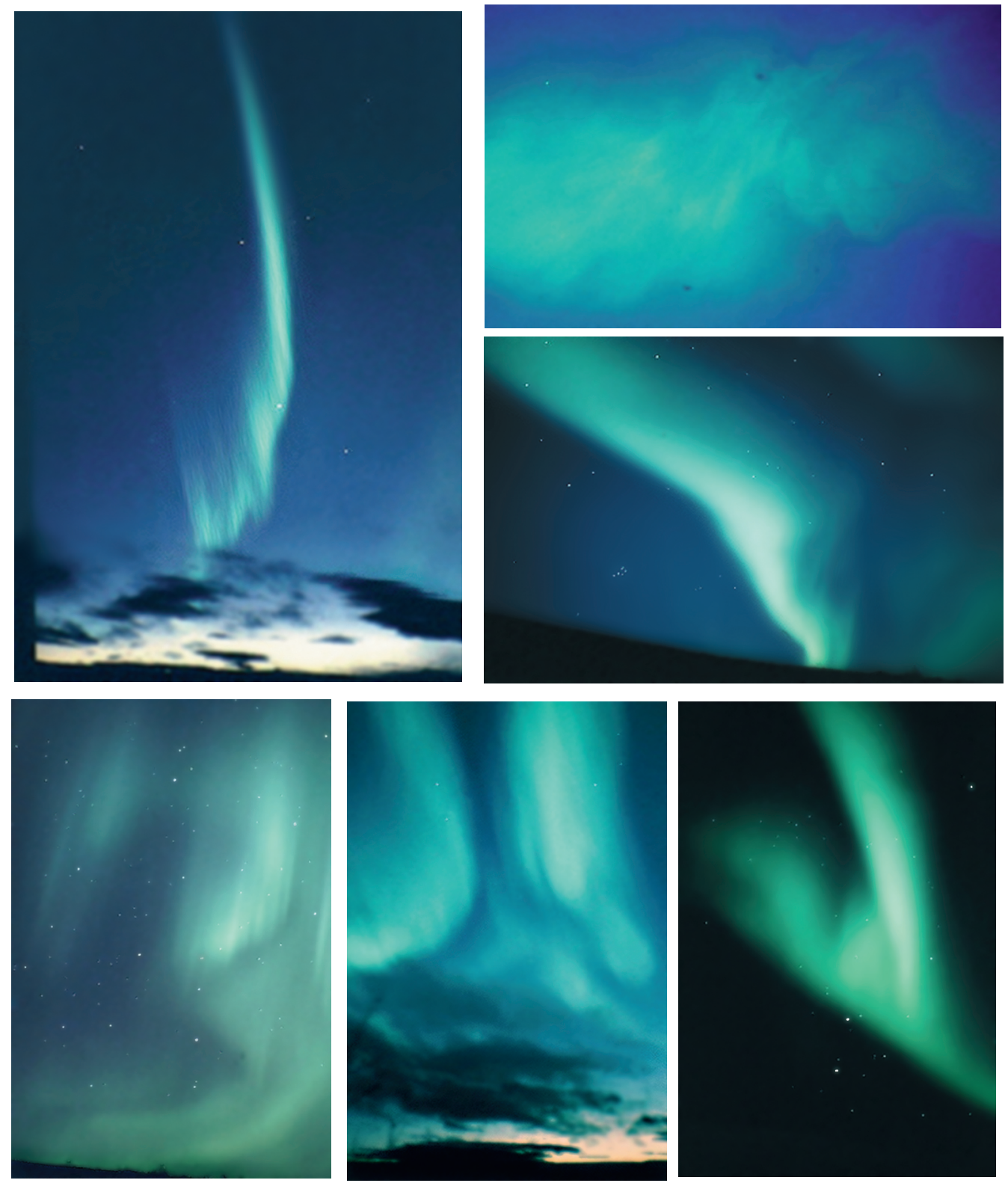

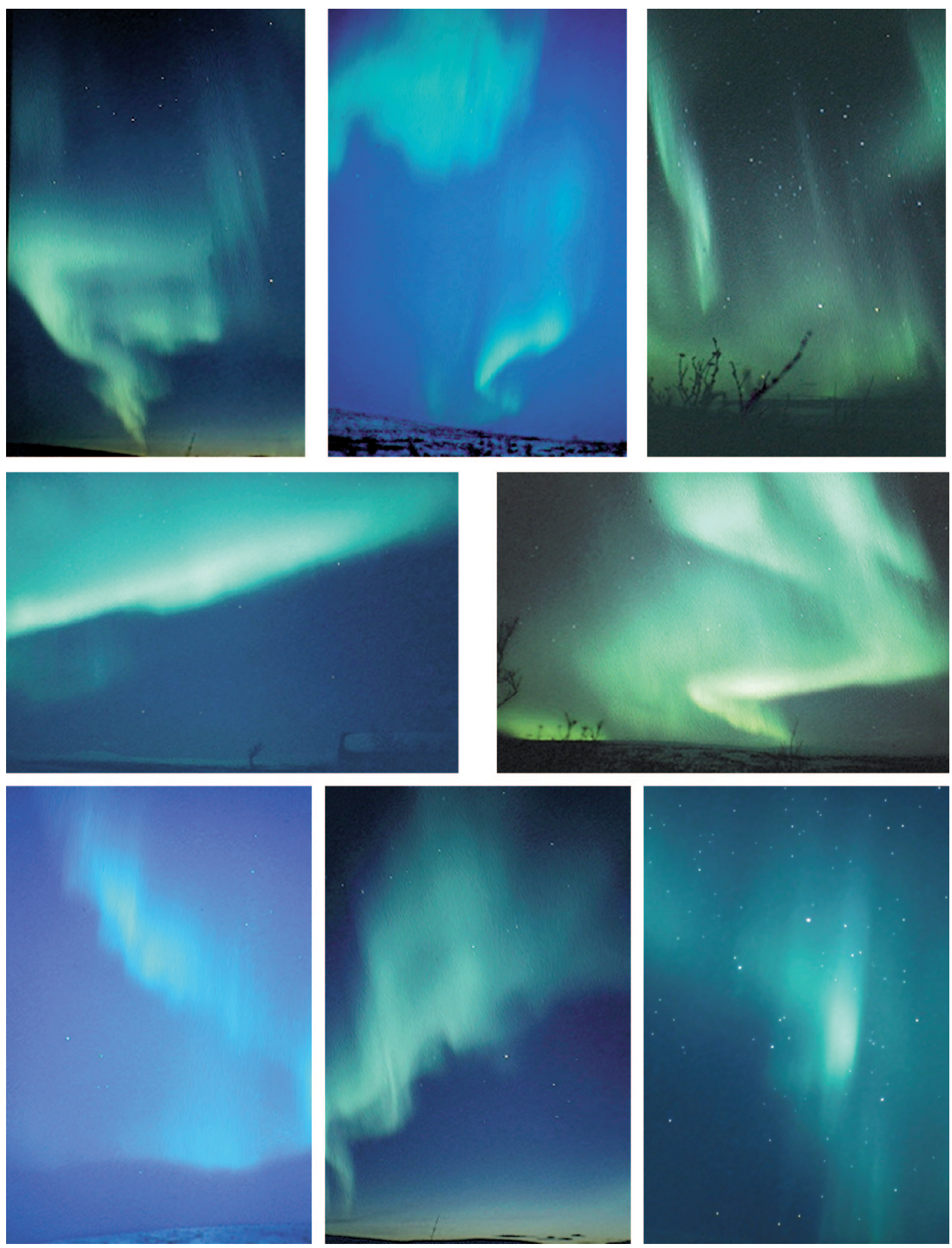
Enfin, Jan Curtis, de l'Alaska Climate Research Center, à Fairbanks en Alaska, a également accepté de montrer quelques-unes de ses œuvres dans ce chapitre.
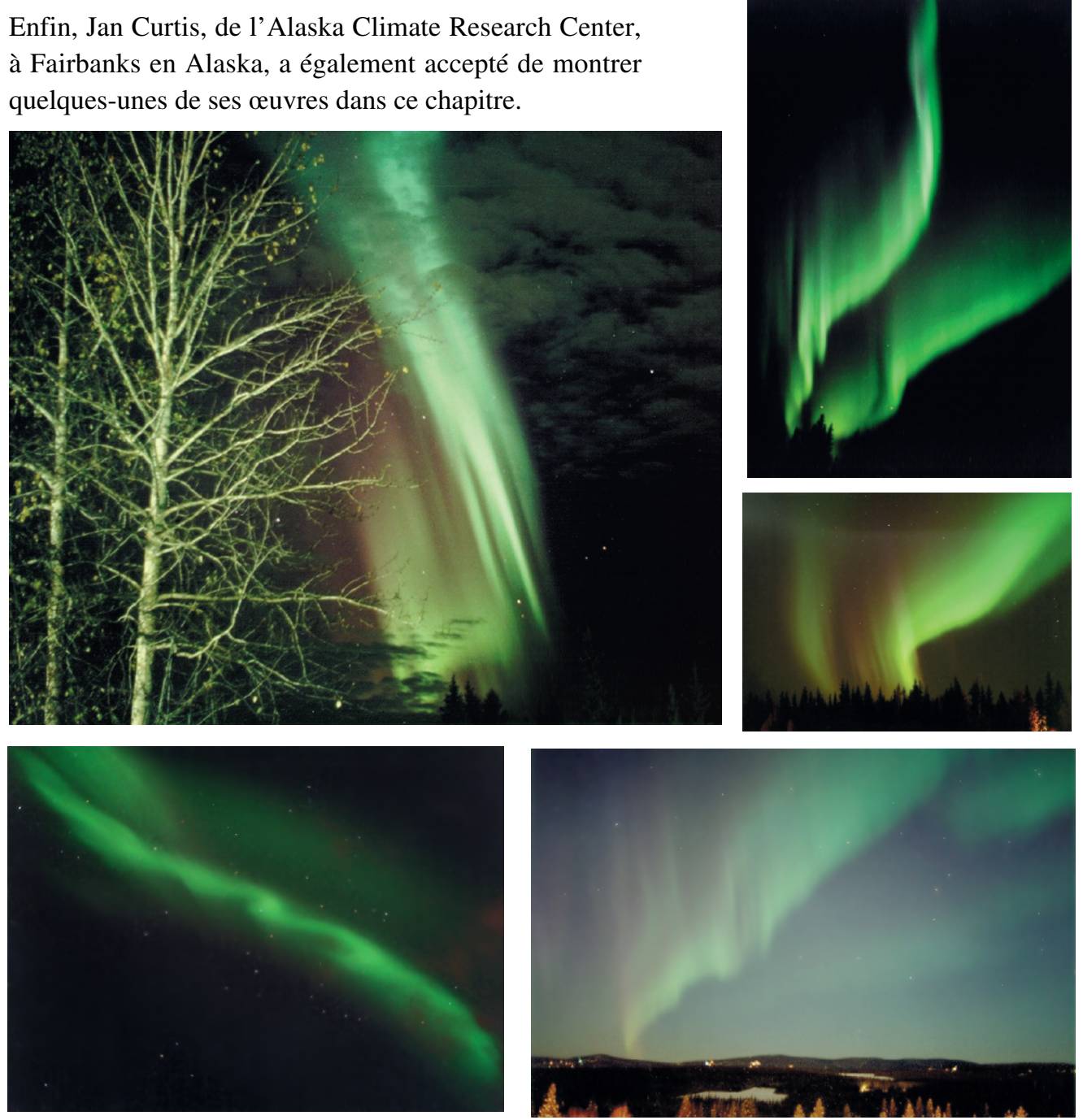

Sur cette dernière photogrpahie, la comète Hale-Bopp survole une aurore diffuse verte.

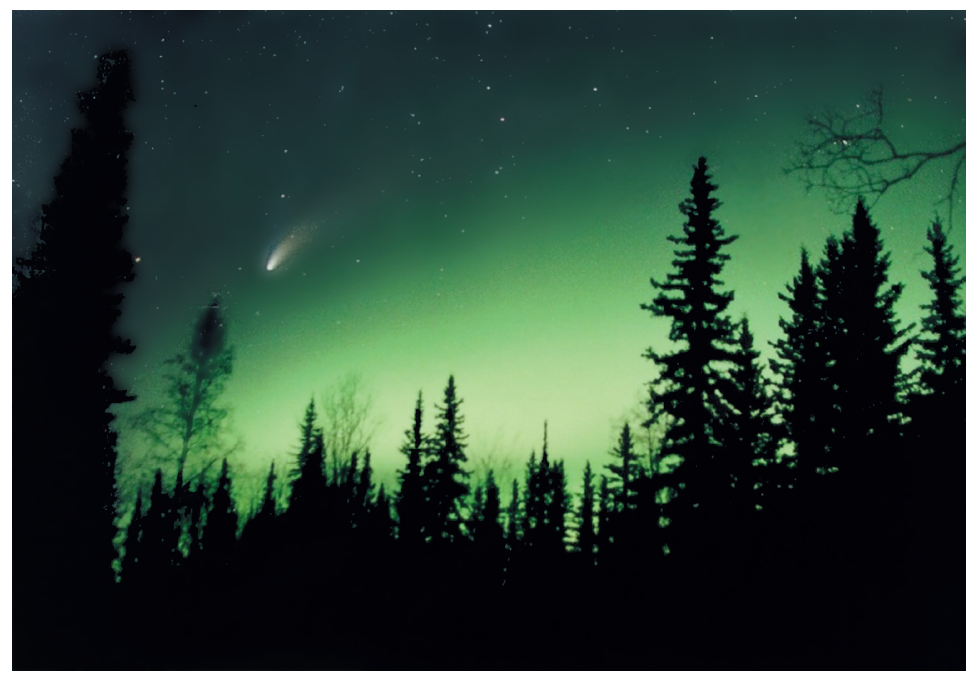




\section{RÉSUMÉ}

Les photons UV, les photoélectrons ou les particules précipitées constituent trois sources d'excitation ou d'ionisation de la thermosphère. Une quatrième source d'excitation est faite de réactions chimiques. Les ions ou atomes excités reviennent à l'état d'équilibre en émettant un rayonnement électromagnétique. Le rayonnement diurne a pour sources principales l'absorption des photons solaire et les réactions chimiques. La nuit, les réactions chimiques constituent la source majeure du rayonnement, mais les précipitations engendrent, en régions aurorale et polaire, un rayonnement intense : les aurores polaires, boréales au nord et australes au sud.

\section{BIBLIOGRAPHIE}

Ce chapitre s'inspire de deux livres remarquables sur le sujet par deux spécialistes mondialement reconnus:

Physics and Chemistry of the upper atmosphere, M.H. Rees, Cambridge University Press, 1989, ISBN 0-521-32305-3.

Ultraviolet spectroscopy and remote sensing of the upper atmosphere, R.R. Meier, Space Science Rev., 58 / 1+2, Kluver academic Publishers, 1991, ISSN 0038-6308.

Le premier ouvrage a déjà été cité. Le chapitre consacré au rayonnement examine les trois spectres longueur d'onde par longueur d'onde. Le choix de R.R. Meier est au contraire d'examiner le rayonnement espèce par espèce. Les références inclues dans ces ouvrages sont très complètes, et le second, totalement consacré au sujet, est très abondamment illustré. Ces ouvrages donnent pour chaque réaction concernée les coefficients de réactions, et le second les discute et en propose souvent toute une palette.

Un troisième auteur a fait un travail extraordinaire dans le domaine. Il s'agit de A.L. Broadfoot. Le spectre diurne de ce chapitre provient de :

$\mathrm{N}_{2}$ triplet band systems and atomic oxygen in the dayglow, A.L. Broadfoot, D.B. Hatfield, E.R. Anderson, T.C. Stone, B.R. Sandel, J.A. Gardner, E. Murad, D.J. Knecht, C.P Pike and R.A. Viereck, Journal Geoph. Res., 102, 11567-11584, 1997.

La discussion des émissions diurne y est d'une grande clarté, et les références les plus récentes sont présentes dans cet article.

Plus ancien, mais encore d'actualité, on pourra lire :

The Optical aurora, de A. Omholt, Springer Verlag, 1971, ISBN 0-387-05486-3.

En particulier, l'auteur y décrit la forme et la morphologie des aurores avec grand détail.

Une autre description d'avant le WEB est particulièrement intéressante à lire, car elle émane de deux des plus grands noms de l'aéronomie. Il s'agit de celle qu'on trouve dans :

Solar-Terrestrial Physics, S.I. Akasofu and S. Chapman, Oxford Univ. Press, 1972. 
Enfin, la bibliographie la plus à jour des réactions conduisant à l'émission des raies rouge ou verte de l'oxygène se trouve dans un article:

Witasse O. et co-auteurs, Annales geophysicae, 1999, sous presse.

Un livre grand public en langue française est à mettre en exergue :

«Les aurores boréales ou les lumières mystérieuses », de Candace Savage, éditions du Trécarré, 1994, ISBN 2-89249-535-0.

La science y est maltraitée, mais on y trouvera de nombreuses croyances et légendes autour des aurores et une relation passionnante des efforts scientifiques pour les comprendre, depuis l'antiquité aux années cinquante.

Sur la toile, on peut trouver facilement de splendides photographies ou animations d'aurores. On y trouve également des descriptifs et une nomenclature qui sortent de l'objet de ce livre. Mes adresses favorites sont :

http://beatles.imv.uit.no/english/science/publicat/waynorth/wn1/contents.htm;http://snake.irf.se/ http://space.sgo.fi/htmls/jpics.html;http://www.ucalgary.ca/ tstronds/satellites/html/isis.html http://ispec.ucsd.edu/virtual/;http://home.sn.no/ kjellr/photo.html http://nis-www.lanl.gov/ mgh/;http://www.geo.mtu.edu/weather/aurora/images/aurora/ http://www.geo.mtu.edu/weather/aurora/images/jan.curtis/ 